\title{
Caracterização dos Cuidadores de Pacientes em Cuidados Paliativos no Domicílio
}

\author{
Characterizing In-Home Caregivers of Patients under Palliative Healthcare \\ Caracterización de los Cuidadores de Pacientes en Cuidados Paliativos en el Hogar
}

\author{
Thaisa Mara de Melo', Inês Gimenes Rodrigues², Denise Rodrigues Costa Schmidt ${ }^{3}$
}

\section{Resumo}

Este estudo teve por objetivo identificar o perfil social dos cuidadores de pacientes oncológicos assistidos pela equipe de Cuidados Paliativos do Sistema de Internação Domiciliar de Londrina (PR), no período de janeiro a julho de 2008, assim como analisar o preparo para o cuidado e as atividades desenvolvidas pelos cuidadores. A amostra constituiu-se de 35 cuidadores e os dados foram processados e analisados no programa Statistical Package for the Social Science (SPSS) version 11.5 for Windows. Entre os cuidadores, 85,7\% eram mulheres, com média de idade de 53 anos. A maioria possuía filhos $(88,6 \%)$; era evangélica $(48,6 \%)$ ou católica $(45,7 \%)$; possuía companheiro $(77,1 \%)$ e ocupação $(60 \%)$; e tinha entre $1-4(37,1 \%)$ ou $9-11(34,3 \%)$ anos de estudo. Tornaramse cuidadores por falta de outra opção $(42,8 \%)$ ou por escolha própria $(40,0 \%)$. Assumiram a função em média há 18 meses. Prestavam cuidados pesados $88,5 \%$, cuidados específicos $80 \%$, e atividades domésticas atreladas aos cuidados $94,2 \%$ dos cuidadores. Sentiam dor no corpo $85,7 \%$ e $45,7 \%$ tinham momentos de recreação. A maioria dos cuidadores manifestou preferência pelo óbito do paciente na residência $(51,4 \%)$. Sentiam-se preparados para cuidar 74,3\% dos cuidadores e para o óbito no domicílio $57,2 \%$. O cuidador domiciliar sente-se preparado para o cuidado e morte no domicílio, apesar de realizar cuidados pesados e específicos, além dos domésticos; sentir dor no corpo e realizar poucas atividades de lazer. Conhecer esse personagem é fundamental para o planejamento de ações e execução de Cuidados Paliativos qualificados no domicílio.

Palavras-chave: Cuidadores; Condiçôes Sociais; Cuidados Paliativos; Assistência Domiciliar; Oncologia; Londrina (PR)

${ }^{1}$ Discente do $4^{\mathrm{o}}$ ano de Enfermagem da Universidade Estadual de Londrina (PR), Brasil.

${ }^{2}$ Enfermeira, docente da Universidade Estadual de Londrina (PR), Brasil. Doutoranda pela Escola de Enfermagem de Ribeirão Preto (EERP)/USP (SP), Brasil.

${ }^{3}$ Doutora em Enfermagem Fundamental pela EERP/USP e Enfermeira da Sala de Recuperação do Hospital Universitário de Londrina (PR), Brasil. Endereço para correspondência: Thaisa Mara de Melo. Rua Professor Samuel Moura, 710 - Apto. 303. Jardim Andrade - Londrina (PR), Brasil CEP: 86061-060. E-mail: thaisamel@yahoo.com.br 


\section{INTRODUÇÃO}

O atual perfil epidemiológico do câncer nos âmbitos mundial e nacional enfatiza a relevância dessa morbidade na sociedade. Esse cenário confirma a importância do câncer como um problema de saúde pública e reforça a necessidade da inserção de Cuidados Paliativos na atenção à saúde do paciente oncológico, considerando que muitos pacientes são diagnosticados em fase avançada e que mais da metade falece em decorrência do câncer. Assim, os serviços de saúde, nesses casos, devem visar não à cura, mas sim à busca por uma melhor qualidade de vida por meio de intervenções que considerem não somente aspectos biológicos, mas também psicológicos, sociais e espirituais dos pacientes $^{1,2}$.

O movimento de Cuidados Paliativos faz-se notório também por buscar a dignidade e a autonomia no processo de morrer. A Organização Mundial da Saúde ${ }^{1}$ descreve Cuidados Paliativos como açóes voltadas aos pacientes que possuem doenças que ameaçam a continuidade da vida e os seus familiares, com o intuito de prevenir e tratar problemas biológicos, psicológicos, sociais e espirituais e, consequentemente, melhorar a qualidade de vida.

Apesar do avanço tecnológico e do aprimoramento do conhecimento médico-científico vivenciados atualmente pela equipe de saúde, nem sempre é possível a obtenção da cura, tornando-se comum nessas situações o uso indiscriminado de tecnologia para prolongar a vida nas realidades hospitalares, mesmo em condições em que a morte é um fator imutável, devido ao esgotamento de todas as possibilidades de cura ${ }^{3}$.

Diante desse cenário, em que muitos pacientes diagnosticados com câncer apresentam-se com doença avançada e incurável, elegível apenas para tratamento paliativo $^{1}$ e, no qual muitos experimentam um prolongamento da vida a qualquer custo pelo entendimento médico da morte como um fracasso, ressalta-se a importância dos Cuidados Paliativos como forma de garantir, a pacientes e familiares, apoio, qualidade de vida e uma morte digna.

Os Cuidados Paliativos podem ser prestados em ambientes de internação hospitalar, ambulatorial e domiciliar. Estima-se que hoje o Brasil possua aproximadamente 40 unidades de Cuidados Paliativos, onde predominam as duas últimas modalidades ${ }^{4}$. Salienta-se que os determinantes do modelo mais apropriado para cada sociedade são as suas próprias necessidades específicas e as particularidades locais dos Serviços de Saúde existentes, assim como de seus provedores ${ }^{4,5}$.
Um estudo ${ }^{6}$ sobre a experiência do serviço de assistência domiciliar de um hospital privado ressaltou a importância de o cuidado domiciliar proporcionar assistência ao cliente, além de capacitar os cuidadores/ familiares para continuidade do cuidado. Enfatiza, ainda, que a assistência domiciliar é uma realidade capaz de auxiliar na transformação da assistência à saúde no país, tornando-a mais eficaz, pois se considera a realidade em que vivem os clientes e suas famílias e, assim, suas reais necessidades.

Para a efetiva implantação e atuação da atenção domiciliar, faz-se necessária a participação do cuidador em conjunto com a equipe de saúde. É considerado cuidador a pessoa responsável por auxiliar o paciente e/ ou idoso dependente de auxílio em suas atividades e rotinas. Em alguns casos, presta os cuidados mediante remuneração, podendo possuir ou não algum vínculo familiar com o paciente ${ }^{7,8}$.

De acordo com Wanderley ${ }^{9}$, o cuidador pode ser classificado em: cuidador familiar: o qual possui algum grau de parentesco com a pessoa cuidada; cuidador terceiro: o qual não possui parentesco com a pessoa cuidada; cuidador leigo: aquele sem qualificação profissional para o exercício legal da atividade de cuidar; cuidador principal: o qual mantém a responsabilidade da pessoa sob seu cuidado de forma permanente; cuidador secundário: aquele que auxilia e/ou substitui o cuidador principal; cuidador remunerado: aquele que recebe remuneração pelo serviço prestado; cuidador voluntário: o qual não recebe remuneração para cuidar do paciente.

Vários estudos que buscam analisar a sobrecarga física, psíquica, social e econômica do cuidador domiciliar têm sido realizados ${ }^{10,11,12,13}$. O portador do câncer terminal, devido ao processo natural da doença, torna-se gradativamente mais dependente de cuidados, necessidade que culmina no surgimento do cuidador domiciliar, predominantemente do sexo feminino e com algum laço familiar com o doente ${ }^{14}$.

O município de Londrina possui desde 1996 um Serviço de Saúde voltado à atenção domiciliar. Criado pela Autarquia Municipal de Saúde, o Sistema de Internação Domiciliar (SID) inicialmente contava com cinco equipes básicas formadas por médicos, enfermeiras e auxiliares de enfermagem e com uma equipe de apoio matricial formada por psicóloga, nutricionista, fisioterapeuta, assistente social e farmacêutica. Em 2005, houve readequações no Serviço que culminaram na reorganização das equipes e na criação da equipe de Cuidados Paliativos Oncológicos ${ }^{15}$.

Um dos critérios para admissão no SID é a presença de um cuidador domiciliar. Essa também é uma 
exigência da Portaria $2.529^{16}$, que aborda a instituição da internação domiciliar no âmbito do Sistema Único de Saúde (SUS) e coloca, como critério de exclusão no Serviço, a ausência de um cuidador contínuo identificado.

O cuidador, portanto, é um importante personagem para a garantia do sucesso do atendimento domiciliar e tem papel fundamental na provisão de cuidados, pois é quem assume e garante a manutenção da assistência necessária ao paciente em seu domicílio.

Frente a essas considerações, surgiu a necessidade de se conhecer o perfil e as atividades dos cuidadores de pacientes em Cuidados Paliativos domiciliares, visando a subsidiar o planejamento e organização de ações mais efetivas por parte das equipes de Cuidados Paliativos. Assim, este estudo procurou elucidar essas questôes por meio dos seguintes objetivos: identificar o perfil social dos cuidadores de pacientes oncológicos em Cuidados Paliativos no domicílio, vinculados ao SID, em Londrina, no período de janeiro a julho de 2008; analisar as atividades dos cuidadores de pacientes em cuidados paliativos no domić́lio e o seu preparo para o cuidado.

\section{MATERIAIS E MÉTODOS}

O presente estudo recebeu aprovação do Comitê de Ética e Pesquisa com Seres Humanos da Universidade Estadual de Londrina (PR)/Hospital Universitário Regional Norte do Paraná sob o parecer 277/07 e foi desenvolvido de acordo com as orientações da Resolução 196/96 do Conselho Nacional de Saúde do Ministério da Saúde ${ }^{17}$.

Trata-se de um estudo de campo, descritivo e transversal realizado no SID do município de Londrina (PR), Brasil. A população do estudo foi composta pelos cuidadores principais de pacientes oncológicos, assistidos pela equipe de Cuidados Paliativos do SID do município de Londrina. Para participar do estudo, os sujeitos deveriam atender aos seguintes critérios de inclusão: ser cuidador domiciliar do paciente que tenha sido assistido pela equipe de Cuidados Paliativos do SID do município de Londrina entre o período de janeiro a julho de 2008; ter idade superior a 18 anos; e aceitar participar do estudo voluntariamente. Nos casos de famílias que possuíam um ou mais cuidadores secundários, optou-se pela aplicação do questionário somente ao cuidador principal'.

Inicialmente, identificou-se que 40 famílias foram atendidas pela equipe no período estabelecido. Após contato com a equipe e cuidadores, três famílias foram excluídas do estudo por não se enquadrarem nos critérios de inclusão e duas por não concordarem em participar do estudo, resultando em uma amostra de 35 cuidadores.

Esses 35 cuidadores foram contatados pela autora via telefone, nos meses de janeiro a agosto de 2008, a qual agendou uma visita no domicílio para a aplicação de um questionário pré-formulado. Nessa ocasião, formalizou-se a participação destes cuidadores no estudo por meio da assinatura do Termo de Consentimento Livre e Esclarecido.

$\mathrm{O}$ instrumento utilizado foi composto por questóes fechadas relacionadas à caracterização do paciente; tempo de acompanhamento do paciente pelo SID; caracterização do cuidador principal; tempo de prestação de cuidados ao paciente; escolha por ser cuidador; cuidados prestados pelo cuidador; preparo do cuidador para o cuidado e para a morte no domicílio. Os dados que não eram do conhecimento do paciente ou cuidador foram obtidos com a equipe do Serviço de Cuidados Paliativos.

Os dados foram processados e analisados no programa Statistical Package for the Social Science (SPSS) version 11.5 for Windows (SPSS). Todas as variáveis sofreram análises descritivas, sendo que as variáveis categóricas foram submetidas à análise de frequência simples, enquanto as contínuas foram analisadas segundo as medidas de tendência central (média e mediana) e dispersão (desvio-padrão). O teste qui-quadrado $\left(\chi^{2}\right)$ foi utilizado para verificar possíveis associações entre as variáveis do estudo. Para tanto, algumas variáveis foram categorizadas para a realização das associações de nosso interesse (tempo de internação no SID e tempo de prestação de cuidados pelo cuidador). O nível de significância adotado foi de igual ou menor que 0,05 .

\section{RESULTADOS}

Conforme se observa nas Tabelas 1 e 2 , a maioria dos cuidadores era do sexo feminino $(85,7 \%)$, com companheiro $(77,1 \%)$. Com relação à escolaridade, as categorias mais frequentes foram de um a quatro anos de estudo $(37,1 \%)$ e de nove a 11 anos $(34,3 \%)$.

A média e a mediana de idade dos cuidadores foi de 53 anos (D.P. $=12,9$ ). Constatou-se, ainda, que 60\% dos cuidadores possuíam alguma ocupação e $40 \%$ não possuíam. Denominaram-se evangélicos $48,6 \%$, católicos $45,7 \%$ e espíritas $5,7 \%$ dos cuidadores.

Do total, 88,6\% dos cuidadores possuíam filhos, com mediana do número de filhos igual a dois, sendo o mínimo de zero e o máximo de nove filhos. Quanto ao grau de parentesco, a maioria dos cuidadores era cônjuges $(\mathrm{n}=13 ; 37,1 \%)$ ou filhos dos pacientes $(\mathrm{n}=10$; $28,6 \%$ ). 
Tabela 1. Distribuição da frequência dos cuidadores de pacientes em Cuidados Paliativos domiciliar, segundo as variáveis sociodemográficas e grau de parentesco com os pacientes. Londrina (PR), Brasil - 2008

\begin{tabular}{|c|c|c|}
\hline Variáveis & $\mathbf{n}$ & $\%$ \\
\hline \multicolumn{3}{|l|}{ Sexo } \\
\hline Feminino & 30 & 85,7 \\
\hline Masculino & 5 & 14,3 \\
\hline \multicolumn{3}{|l|}{ Estado civil } \\
\hline Com companheiro & 27 & 77,1 \\
\hline Sem companheiro & 8 & 22,9 \\
\hline \multicolumn{3}{|l|}{ Escolaridade } \\
\hline Nenhuma & 1 & 2,9 \\
\hline $1-4$ anos & 13 & 37,1 \\
\hline $5-8$ anos & 7 & 20,0 \\
\hline $9-11$ anos & 12 & 34,3 \\
\hline$>12$ anos & 2 & 5,7 \\
\hline \multicolumn{3}{|l|}{ Filhos } \\
\hline Sim & 31 & 88,6 \\
\hline Não & 4 & 11,4 \\
\hline \multicolumn{3}{|l|}{ Ocupação } \\
\hline Com ocupação & 21 & 60,0 \\
\hline Sem ocupação & 14 & 40,0 \\
\hline \multicolumn{3}{|l|}{ Religião } \\
\hline Evangélico & 17 & 48,6 \\
\hline Católico & 16 & 45,7 \\
\hline Espírita & 2 & 5,7 \\
\hline \multicolumn{3}{|l|}{ Grau de parentesco } \\
\hline Cônjuge & 13 & 37,1 \\
\hline Filho & 10 & 28,6 \\
\hline Parente distante & 6 & 17,1 \\
\hline Irmão & 3 & 8,6 \\
\hline Sem parentesco & 2 & 5,7 \\
\hline Pai/mãe & 1 & 2,9 \\
\hline Total & 35 & 100 \\
\hline
\end{tabular}

A média de tempo transcorrido desde quando os cuidadores assumiram esse papel até a data da entrevista foi de aproximadamente 18 meses (D.P.= 30,7), mediana de 12 meses, com intervalo de tempo que variou entre um a 180 meses (Tabela 2). Do total ( $\mathrm{n}=35), 40,0 \%$ $(\mathrm{n}=14)$ dos cuidadores prestavam cuidados há 7-12 meses e 34,3\% ( $\mathrm{n}=12)$ há mais de 13 meses.
$\mathrm{Na}$ Tabela 3, observa-se discreta diferença entre as porcentagens dos que afirmaram ter momentos de lazer $(45,7 \%)$ e dos que referiram não possuir tais atividades $(54,3 \%)$. Quando perguntado sobre a presença de dor no corpo, $85,7 \%$ dos cuidadores responderam que sentiam dor.

Verificou-se, ainda, na Tabela 3, que 74,3\% dos cuidadores de pacientes em Cuidados Paliativos no domicílio afirmaram sentir-se preparados para cuidar do paciente no domicílio. Apenas $17,1 \%$ dos cuidadores relataram não estar preparados para as atividades e 8,6\% relativamente preparados para essa situação. Ao analisar uma possível relação entre essa variável e o tempo de acompanhamento com a equipe do SID, não se encontrou resultado estatisticamente significante $(\mathrm{p}=0,36)$.

Tabela 3. Distribuição da frequência dos cuidadores, segundo a realização de atividades de lazer, presença de dores no corpo e preparo para o cuidado. Londrina (PR), Brasil - 2008

\begin{tabular}{l|c|c}
\hline \multicolumn{1}{c|}{ Variáveis } & $\mathbf{n}$ & $\%$ \\
\hline Atividades de lazer & 19 & 54,3 \\
$\quad$ Não & 16 & 45,7 \\
Sim & & \\
Dores no corpo & 30 & 85,7 \\
$\quad$ Sim & 5 & 14,3 \\
Não & & \\
Preparo para cuidar do & & \\
paciente no domicílio & 26 & 74,3 \\
Sim & 6 & 17,1 \\
Não & 3 & 8,6 \\
$\quad$ Relativamente & 35 & 100 \\
\hline Total & & \\
\hline
\end{tabular}

Conforme apresentado na Tabela 4, constatou-se que o tempo transcorrido desde que o cuidador assumiu os cuidados ao paciente $(\mathrm{p}=0,453)$, assim como possuir alguma ocupação $(\mathrm{p}=0,324)$, não apresentou resultado estatisticamente significante com a presença de dor no corpo, embora a maioria $(85,7 \%)$ dos cuidadores tenha referido presença de dor no corpo para realização dos cuidados aos pacientes.

Tabela 2. Estatística descritiva da idade, número de filhos e tempo de cuidados prestados ao paciente entre os cuidadores de pacientes em Cuidados Paliativos domiciliar. Londrina (PR), Brasil - 2008

\begin{tabular}{l|c|c|c}
\hline \multicolumn{1}{c|}{ Variáveis } & Média (D.P.)* & Mediana & Intervalo \\
\hline Idade (em anos) & $53(12,9)$ & 53 & $23-81$ \\
Filhos & $3(1,7)$ & 2 & $0-9$ \\
Tempo (em meses) de cuidados prestados ao paciente & $18(30,7)$ & 12 & $1-180$ \\
\hline
\end{tabular}

* Desvio-padrão 
Tabela 4. Distribuição da frequência de dor no corpo entre os cuidadores, segundo o tempo de cuidados prestados ao paciente e ocupação do cuidador. Londrina (PR), Brasil - 2008

\begin{tabular}{|c|c|c|c|c|c|c|c|}
\hline \multirow{3}{*}{ Variáveis } & \multicolumn{4}{|c|}{$\begin{array}{c}\text { Dor no corpo para realizar os } \\
\text { cuidados }\end{array}$} & \multirow{2}{*}{\multicolumn{2}{|c|}{ Total }} & \multirow[t]{3}{*}{$\mathbf{p}^{*}$} \\
\hline & \multicolumn{2}{|c|}{ Sim } & \multicolumn{2}{|c|}{ Não } & & & \\
\hline & $\mathbf{n}$ & $\%$ & $\mathbf{n}$ & $\%$ & $\mathbf{n}$ & $\%$ & \\
\hline $\begin{array}{l}\text { Tempo (em meses) de cuidados } \\
\text { prestados ao paciente }\end{array}$ & & & & & & & 0,453 \\
\hline 1 a 3 & 4 & 66,7 & 2 & 33,3 & 6 & 100,0 & \\
\hline 4 a 6 & 3 & 100,0 & 0 & 0 & 3 & 100,0 & \\
\hline 7 a 12 & 12 & 85,7 & 2 & 14,3 & 14 & 100,0 & \\
\hline 13 ou mais & 11 & 91,7 & 1 & 8,3 & 12 & 100,0 & \\
\hline Ocupação do cuidador & & & & & & & 0,324 \\
\hline Possui ocupação & 11 & 78,6 & 3 & 21,4 & 14 & 100,0 & \\
\hline Não possui ocupação & 19 & 90,5 & 2 & 9,5 & 21 & 100,0 & \\
\hline Total & 30 & 85,7 & 5 & 14,3 & 35 & 100,0 & \\
\hline
\end{tabular}

$\mathrm{Na}$ Tabela 5, foram verificados os fatores que motivaram os cuidadores de pacientes em Cuidados Paliativos no domicílio a se tornarem cuidadores principais, sendo que a maioria assume essa função por não ter outra opção $(42,8 \%)$ ou por escolha própria $(40,0 \%)$.

Tabela 5. Distribuição dos fatores que motivaram os cuidadores principais de pacientes oncológicos em Cuidados Paliativos no domicílio a assumirem esse papel. Londrina (PR), 2008

\begin{tabular}{l|c|c}
\hline Motivação para o cuidado & $\mathbf{n}$ & $\%$ \\
\hline Por não ter outra opção & 15 & 42,8 \\
Escolha própria & 14 & 40,0 \\
Consenso familiar & 5 & 14,3 \\
Escolha do paciente & 1 & 2,9 \\
\hline Total & $\mathbf{3 5}$ & $\mathbf{1 0 0}$ \\
\hline
\end{tabular}

Em relação aos cuidados prestados pelo cuidador, classificou-se como "cuidados pesados" aqueles prestados a pacientes com maior grau de dependência, como dar o banho, vestir o paciente, deitar e levantar da cama, administrar os medicamentos, dar os alimentos, movimentar o paciente no leito e realizar higiene íntima após eliminações. "Cuidados leves" foram considerados os prestados a pacientes com pequeno grau de dependência, como auxiliar no banho e no vestir, auxiliar o paciente a deitar e levantar da cama, auxiliar na medicação e nas refeiçôes.

Denominou-se "cuidados específicos" os que exigiam o aprendizado de técnicas por parte do cuidador, por exemplo, os cuidados relacionados às sondas nasogástrica, nasoenteral e vesical, traqueostomia, aspiração, ostomias intestinais, curativos e hipodermóclise. Utilizou-se o termo "transporte" quando os cuidadores levavam os pacientes para locais fora do domicílio para realização de exames, quimioterapia, radioterapia, fisioterapia, entre outros.

Conforme observado na Tabela 6, grande parte dos cuidadores de pacientes em Cuidados Paliativos no domicílio prestava, ao paciente, cuidados pesados $(88,5 \%)$ e específicos $(80,0 \%)$, sendo também responsável pelo seu transporte para locais fora do domicílio (88,5\%). Prestavam cuidados leves $42,8 \%$ dos cuidadores e, ainda, $34,2 \%$ prestavam tanto cuidados leves quanto pesados.

Tabela 6. Distribuição de frequência dos cuidados prestados e das atividades realizadas pelo cuidador de paciente em Cuidados Paliativos no domicílio. Londrina (PR), Brasil - 2008

\begin{tabular}{l|c|c}
\hline \multicolumn{1}{c|}{ Variáveis } & $\mathbf{n}$ & $\%$ \\
\hline Cuidados pesados & 31 & 88,5 \\
Cuidados leves & 15 & 42,8 \\
Cuidados pesados e leves & 12 & 34,2 \\
Cuidados específicos & 28 & 80,0 \\
Transporte & 31 & 88,5 \\
Atividades domésticas & 33 & 94,2 \\
Atividades fora do domicílio & 20 & 57,1 \\
Cuidados com outros & 15 & 42,8 \\
\hline
\end{tabular}

Com relação às outras atividades do dia-a-dia que também eram responsabilidades do cuidador, classificou-se como "domésticas" (cuidados com roupas, com a casa e preparo dos alimentos), "cuidados com 
outros" (cuidados com crianças ou outros idosos). Pagamentos de contas e compras foram classificados como "atividades fora do domicílio". Identificou-se que 94,2\% dos cuidadores realizavam alguma atividade doméstica. Grande parte dos cuidadores era responsável por cuidados com crianças ou outros idosos $(42,8 \%)$ e a maioria por atividades fora do domicílio $(57,1 \%)$.

Com relação à preferência pelo local do óbito do paciente, $51,4 \%$ dos cuidadores manifestaram preferência pelo óbito no domicílio. Os que colocaram nunca ter refletido sobre o assunto representaram $25,7 \%$, e os que preferiam que o óbito ocorresse no hospital, 22,9\% (Tabela 7). Ao analisar uma possível relação entre a variável preferência pelo local do óbito do paciente e o tempo de acompanhamento pela equipe do serviço, encontrou-se resultado estatisticamente não significante, contrariando nossa percepção inicial de que essas variáveis possuíam relação entre si $(\mathrm{p}=0,73)$.

Ainda na Tabela 7, apesar de não ter encontrado resultado estatisticamente significante entre as variáveis religião do cuidador e preferência deste pelo local do óbito do paciente $(\mathrm{p}=0,40)$, observou-se que os cuidadores espíritas $(\mathrm{n}=2 ; 100 \%)$ e a maioria dos evangélicos $(n=10 ; 58,8 \%)$ preferem que o óbito do paciente ocorra no domicílio. Entre os cuidadores católicos, observa-se a mesma frequência entre os que preferem a ocorrência do óbito no domicílio e os que nunca refletiram sobre o assunto.

Com relação ao preparo do cuidador para a ocorrência da morte do paciente no domicílio, 57,2\% afirmaram sentir-se preparados para essa situação, $31,4 \%$ despreparados e $11,4 \%$ relativamente preparados. Ao analisar relação dessa variável com a variável religião do cuidador, verificou-se um resultado estatisticamente não significante $(\mathrm{p}=0,44)$ (Tabela 7$)$.

Com relação às características dos pacientes em Cuidados Paliativos vinculados ao SID (Tabelas 8 e 9) e atendidos pelos cuidadores que participaram do estudo, constatou-se que a maioria era do sexo masculino $(57,1 \%)$, com companheiro $(68,6 \%)$, e média de idade de 66,7 anos (D.P.= 14,6), mediana de 67 anos. Quanto à escolaridade, a maioria (42,9\%) obteve de um a quatro anos de estudo, enquanto $20,0 \%$ estudaram de cinco a oito anos. A média de tempo de acompanhamento desses pacientes com a equipe do SID foi de 85 dias (D.P.= $89,9)$ e a mediana de 50 dias. O tempo mínimo de acompanhamento com a equipe foi de quatro dias e o máximo, de 394 dias.

Haviam sido esclarecidos sobre seu estado de saúde $42,9 \%$ dos pacientes; porém, ao analisar a relação entre essa variável e o tempo de acompanhamento pela equipe do Serviço, observou-se um resultado estatisticamente não significante $(\mathrm{p}=0,37)$.

De acordo com os dados coletados com os cuidadores, $31,4 \%(\mathrm{n}=11)$ dos pacientes manifestaram em algum momento preferência pela ocorrência de seu óbito no domicílio e 5,7\% (n=2) declararam preferência pelo óbito no ambiente hospitalar. A maioria dos pacientes, $57,2 \%(n=20)$, segundo o cuidador, nunca falou sobre o assunto. Foram consultados os cuidadores de dois pacientes que estavam mentalmente impossibilitados de manifestar suas opiniōes por apresentarem alteração do nível de consciência. Considerou-se a questão como "não se aplica" (5,7\%), pois os pacientes encontravam-se nessa situação antes mesmo da elucidação do diagnóstico e encaminhamento

Tabela 7. Distribuição de frequência do cuidador, segundo sua religião, preferência pelo local do óbito do paciente e preparo para o óbito do paciente no domicílio. Londrina (PR), Brasil - 2008

\begin{tabular}{|c|c|c|c|c|c|c|c|c|c|}
\hline \multirow{2}{*}{ Variáveis } & \multicolumn{6}{|c|}{ Religião do cuidador } & \multirow{2}{*}{\multicolumn{2}{|c|}{ Total }} & \multirow{2}{*}{$\mathbf{p}^{*}$} \\
\hline & \multicolumn{2}{|c|}{ Evangélico } & \multicolumn{2}{|c|}{ Católico } & \multicolumn{2}{|c|}{ Espírita } & & & \\
\hline $\begin{array}{l}\text { Preferência do cuidador pelo } \\
\text { local do óbito do paciente }\end{array}$ & $\mathbf{n}$ & $\%$ & $\mathbf{n}$ & $\%$ & $\mathbf{n}$ & $\%$ & $\mathbf{n}$ & $\%$ & 0,40 \\
\hline No domicílio & 10 & 58,8 & 6 & 37,5 & 2 & 100,0 & 18 & 51,4 & \\
\hline No hospital & 4 & 23,5 & 4 & 25,0 & 0 & 0 & 8 & 22,9 & \\
\hline Não refletiu sobre o assunto & 3 & 17,7 & 6 & 37,5 & 0 & 0 & 9 & 25,7 & \\
\hline $\begin{array}{l}\text { Preparo do cuidador para o } \\
\text { óbito do paciente no domicílio }\end{array}$ & & & & & & & & & 0,44 \\
\hline Preparado & 10 & 58,8 & 8 & 50,0 & 2 & 100,0 & 20 & 57,2 & \\
\hline Despreparado & 4 & 23,5 & 7 & 43,7 & 0 & 0 & 11 & 31,4 & \\
\hline Relativamente preparado & 3 & 17,7 & 1 & 6,3 & 0 & 0 & 4 & 11,4 & \\
\hline Total & 17 & 100 & 16 & 100 & 2 & 100 & 35 & 100 & \\
\hline
\end{tabular}

${ }^{*} \chi^{2}$ 
para a equipe de Cuidados Paliativos, segundo seus cuidadores.

Ao analisar a relação existente entre a preferência do paciente pelo local de seu óbito e a consciência de seu diagnóstico/prognóstico, observou-se que saber o diagnóstico não interferiu na escolha do paciente $(\mathrm{p}=0,07)$. Também não se observou relação estatisticamente significante entre o tempo de acompanhamento da equipe e a preferência do paciente pelo local de seu óbito $(\mathrm{p}=0,59)$.

Tabela 8. Distribuição da frequência dos pacientes em Cuidados Paliativos domiciliar segundo as variáveis sociodemográficas, esclarecimento sobre o estado de saúde e preferência pelo local de óbito. Londrina (PR), Brasil - 2008

\begin{tabular}{|c|c|c|}
\hline Variáveis & $\mathrm{n}$ & $\%$ \\
\hline \multicolumn{3}{|l|}{ Sexo } \\
\hline Masculino & 20 & 57,1 \\
\hline Feminino & 15 & 42,9 \\
\hline \multicolumn{3}{|l|}{ Estado civil } \\
\hline Com companheiro & 24 & 68,6 \\
\hline Sem companheiro & 11 & 31,4 \\
\hline \multicolumn{3}{|l|}{ Escolaridade } \\
\hline Nenhuma & 6 & 17,1 \\
\hline $1-4$ anos & 15 & 42,9 \\
\hline $5-8$ anos & 7 & 20,0 \\
\hline 9-11 anos & 5 & 14,3 \\
\hline$>12$ anos & 2 & 5,7 \\
\hline \multicolumn{3}{|l|}{$\begin{array}{l}\text { Esclarecimento sobre seu } \\
\text { estado de saúde }\end{array}$} \\
\hline Não & 18 & 51,4 \\
\hline Sim & 15 & 42,9 \\
\hline Não se aplica & 2 & 5,7 \\
\hline \multicolumn{3}{|l|}{$\begin{array}{l}\text { Preferência pelo local de } \\
\text { seu óbito }\end{array}$} \\
\hline Não falou sobre o assunto & 20 & 57,2 \\
\hline No domicílio & 11 & 31,4 \\
\hline No hospital & 2 & 5,7 \\
\hline Não se aplica & 2 & 5,7 \\
\hline Total & 35 & 100 \\
\hline
\end{tabular}

Tabela 9. Estatística descritiva da idade e tempo de acompanhamento no SID dos pacientes em Cuidados Paliativos. Londrina (PR), Brasil - 2008

\begin{tabular}{l|c|c|c}
\hline \multicolumn{1}{c|}{ Variáveis } & $\begin{array}{c}\text { Média } \\
\text { (D.P.) }\end{array}$ & Mediana & Intervalo \\
\hline Idade (em anos) & $\begin{array}{c}66,7 \\
(14,6)\end{array}$ & 67 & $31-88$ \\
$\begin{array}{l}\text { Tempo de } \\
\begin{array}{l}\text { acompanhamento } \\
\text { no SID (em dias) }\end{array}\end{array}$ & $\begin{array}{c}85,0 \\
(89,9)\end{array}$ & 50 & $4-394$ \\
\hline
\end{tabular}

* Desvio-padrão

\section{DISCUSSÃO}

Neste estudo, verifica-se o predomínio do sexo feminino $(85,7 \%)$ nas atividades de cuidador de pacientes em Cuidados Paliativos assistidos no domicílio. A média de idade dos cuidadores observada foi de 53 anos, com escolaridade de um a quatro anos $(37,1 \%)$. Referiram possuir alguma ocupação $60 \%$ dos cuidadores. As religiōes predominantes entre os cuidadores foram a evangélica $(48,6 \%)$ e a católica $(45,7 \%)$. Com relação à motivação do cuidador para o cuidado, constatou-se que essa não se dá ao acaso, pois na grande maioria das vezes ocorre por falta de outra opção $(42,8 \%)$, ou por escolha do próprio cuidador $(40,0 \%)$.

A partir de uma revisão bibliográfica ${ }^{11}$, Floriani e Schramm também verificaram que o cuidador informal de idosos costuma ser do sexo feminino, com idade avançada e, na maioria das vezes, filho ou cônjuge do paciente, dividindo as atividades relacionadas ao cuidado com os afazeres domésticos.

Ao caracterizar cuidadores de idosos dependentes, Almeida ${ }^{18}$ constatou o predomínio do sexo feminino entre os cuidadores $(94,2 \%)$ e que $65,4 \%$ dos cuidadores possuíam companheiro, sendo a maioria filho $(50,0 \%)$ ou cônjuge $(28,0 \%)$ do paciente. Com relação à idade do cuidador, 26,9\% possuíam entre 50-59 anos e 30,8\% entre 60-69 anos. Diferindo do observado no presente estudo, 90,4\% dos cuidadores não tinham ocupação. As atividades domésticas não deixaram de ser realizadas pelo cuidador e $67,3 \%$ dos cuidadores afirmaram sentir dores no corpo, assemelhando-se aos resultados encontrados neste estudo.

Uma pesquisa ${ }^{19}$ sobre cuidadores domiciliares de pessoas incapacitadas por acidente vascular cerebral (AVC) também confirma o predomínio do sexo feminino entre os cuidadores $(77,1 \%)$ que possuíam entre 40-59 anos (54,3\%). Afirmaram ter companheiro $74,3 \%$ dos cuidadores. Eram católicos ou evangélicos $88,6 \%$ dos cuidadores de pacientes com AVC. Em relação ao grau de parentesco, houve predomínio de cuidadores filhos $(40,0 \%)$ e cônjuges $(22,9 \%)$ de pacientes, resultado semelhante ao de nosso estudo. Ao contrário do observado no presente estudo, $42,9 \%$ dos cuidadores possuíam alguma ocupação e a maioria $(37,1 \%)$ havia estudado entre cinco e oito anos.

Em uma abordagem qualitativa, Silva e Acker ${ }^{13}$ também confirmaram o predomínio do sexo feminino entre os cuidadores de pacientes oncológicos, na qual 100\% (8) eram mulheres. Também confirmaram o predomínio das religiōes católicas e evangélicas entre os sujeitos do estudo. A média de idade observada foi de 43 anos e $75 \%$ das cuidadoras eram cônjuges ou 
filhas dos pacientes. A maioria das cuidadoras possuía o ensino fundamental. Ainda, constataram a existência de motivações pessoais ou familiares para o cuidado e que as cuidadoras exerciam atividades domésticas atreladas às atividades de cuidador.

Floriani ${ }^{10}$ constatou que os cuidados geralmente são assumidos por mulheres adultas e idosas e que a proximidade, seja parental, física ou afetiva, costuma influenciar a escolha em ser cuidador. Assim, o papel do cuidador é fortemente influenciado pelo grau de parentesco e pela relação de gênero?.

Um estudo ${ }^{20}$ sobre pacientes encaminhados para atendimento paliativo domiciliar também constatou a relação familiar entre cuidadores e pacientes em $92 \%$ dos casos.

Por ser um papel histórico, cultural e socialmente atrelado às mulheres, além de ser visto como uma extensão das atividades domésticas, frequentemente cabe à figura feminina assumir os cuidados daquele que necessita ${ }^{9,18}$.

No que se refere ao preparo do cuidador para a prestação de cuidados no domicílio, a grande maioria se sente preparada, não sendo observada relação entre o tempo de acompanhamento com a equipe e a percepção do cuidador sobre seu preparo $(\mathrm{p}=0,61)$. Todavia um estudo de abordagem qualitativa ${ }^{21}$ acrescenta que a atuação da equipe determina o desenvolvimento da segurança do cuidador sobre como cuidar no domicílio.

A análise dos cuidados prestados pelo cuidador comprova que a maioria deles realiza atividades pesadas $(88,5 \%)$ e complexas $(80,0 \%)$. Essa característica também foi observada por Alemida ${ }^{18}$, em que grande parte dos cuidadores realizam cuidados pesados que exigem condicionamento físico, como a vestimenta $(51,9 \%)$ e o banho do paciente $(42,3 \%)$. O fato de conciliar as atividades domésticas aos cuidados $(94,2 \%)$, já apontado na discussão anteriormente, também foi observado em outros estudos ${ }^{11,13,18}$.

Analisando essa sobrecarga vivenciada pelo cuidador domiciliar, a dor no corpo relatada por estes $(85,7 \%)$ também foi uma característica observada por Almeida ${ }^{18}$ $(67,3 \%)$.

Constata-se que a maioria dos cuidadores prefere que óbito do paciente ocorra no domicílio, sentindo-se preparados para essa situação. Floriani ${ }^{10}$ reflete sobre a ocorrência do óbito no domicílio e questiona a utilização desse acontecimento como parâmetro de qualidade dos serviços de internaçôes domiciliares, pois diferentes culturas e experiências de vida fazem com que algumas famílias não se sintam preparadas para que esse momento ocorra em casa. Dessa forma, ressalta-se que é necessário considerar não o local da morte, mas a forma como ela ocorre ${ }^{11}$.

Ressalta-se o escasso número de estudos quantitativos identificados sobre o tema em nosso meio após extenso levantamento bibliográfico. Para que se possa planejar e adequar açóes de atenção ao cuidador de acordo com diferentes culturas e realidades, propõe-se a realização de mais estudos sobre o cuidador de pacientes em Cuidados Paliativos no domicílio, área na qual há muitos aspectos a serem explorados.

\section{CONCLUSÃO}

O estudo reflete sobre a relevância do cuidador domiciliar na provisão de cuidados ao paciente em seu domicílio e enfatiza a necessidade de se conhecer esse personagem que assume e garante a manutenção de uma assistência de qualidade ao paciente em Cuidados Paliativos.

Conhecer os cuidadores domiciliares é fundamental para a garantia de uma assistência de qualidade ao paciente em Cuidados Paliativos na residência, pois são estes os responsáveis por colocar em prática todo o plano assistencial elaborado pela equipe.

Com o acompanhamento da equipe de Cuidados Paliativos do SID, o cuidador, em sua maioria, sentiuse preparado para o cuidado e para a morte no domicílio, mesmo assumindo essa função por não ter outra opção; realizando cuidados pesados e específicos, além dos domésticos; sentindo dor no corpo e realizando poucas atividades de lazer.

Ainda, observa-se, na prática, idosos cuidando de idosos, situação que justifica grande parte das dificuldades encontradas pelo cuidador na prestação de cuidados e que pode gerar complicação de sua saúde pela frequente presença de comorbidades associadas à idade avançada.

Por conseguinte, é fundamental a atuação da equipe junto ao cuidador não somente no sentido de educá-lo para o cuidado. Necessita-se compreender as mudanças que ocorrem em sua vida; o impacto tanto físico quanto social e emocional, assim como a sobrecarga, acarretados pela responsabilidade de cuidar. Assim, conhecendo essas questôes relacionadas ao cuidador e aos cuidados no domicílio, torna-se possível o planejamento de estratégias por parte das equipes domiciliares de Cuidados Paliativos que facilitem o dia-a-dia, diminuam a sobrecarga e, consequentemente, melhorem a qualidade de vida desses cuidadores.

Declaração de Conflito de Interesses: Nada a Declarar. 


\section{REFERÊNCIAS}

1. World Health Organization. National cancer control programmes: policies and managerial guidelines. 2 ed. Geneva: World Health Organization; 2002.

2. Pereira MT, Reis TCR. A não-ressucitação, do ponto de vista do médico em uma unidade de cuidados paliativos oncológicos. Revista brasileira de cancerologia 2007; 53(2):225-9.

3. Kovács MJ. Bioética nas questôes da vida e da morte. Psicologia-USP 2003;14(2):115-67.

4. Maciel MGS, Rodrigues LF, Naylor C, Bettega R, Barbosa SM, Burlá C, et al. Academia Nacional de Cuidados Paliativos. Critérios de qualidade para os cuidados paliativos no Brasil. Rio de Janeiro: Diagraphic; 2006.

5. Silva RCF, Hortale VA. Cuidados paliativos oncológicos: elementos para o debate de diretrizes nesta área. Cad Saude Publica 2006;22(10):2055-66.

6. Fabrício SCC, Wehbe G, Nassur FB, Andrade JI. Assistência domiciliar: a experiência de um hospital privado do interior paulista. Rev Lat Am Enfermagem 2004;12(5):721-6.

7. Ministério da Saúde (Brasil). Portaria no 2.528, de 19 de outubro de 2006. Dispõe sobre a Política Nacional de Saúde da Pessoa Idosa. Brasília (DF): Ministério da Saúde; 2006.

8. Ministério da Saúde (Brasil). Resolução da Diretoria Colegiada - RDC no 11, de 26 de janeiro de 2006. Dispóe sobre o Regulamento Técnico de Funcionamento de Serviços que prestam Atenção Domiciliar. Brasília (DF): Ministério da Saúde; 2006.

9. Wanderley MB, Blanes DN, Arregul CC, Sanda CT, Beltrão REV. Publicização do papel do cuidador domiciliar. São Paulo: IEE/PUC-SP; Brasília: Secretaria de Assistência Social/ MPAS; 1998.

10. Floriani CA. Cuidador familiar: sobrecarga e proteção. Revista brasileira de cancerologia 2004;50(4):341-5.

11. Floriani CA, Schramm FR. Atendimento domiciliar ao idoso: problema ou solução? Cad Saude Publica 2004;20(4):986-94.
12. Floriani CA, Schramm FR. Cuidador do idoso com câncer avançado: um ator vulnerado. Cad Saude Publica 2006; 22(3):527-34.

13. Silva CAM, Acker JIBV. O cuidado paliativo domiciliar sob a ótica de familiares responsáveis pela pessoa portadora de neoplasia. Rev Bras Enferm 2007;60(2):150-4.

14. Py L. Cuidar do cuidador: transbordamento e carência. Revista brasileira de cancerologia 2004;50(4):346-50.

15. Feuerwerker LCM. A atenção domiciliar no município de Londrina [série on line] 2007 [citado em 2008 Out 18]. Disponível em: URL: http://www.hucff.ufrj.br/ micropolitica/pesquisas/atencaodomiciliar/textos/ londrina.pdf

16. Ministério da Saúde (Brasil). Portaria no 2.529, de 19 de outubro de 2006. Dispõe sobre as diretrizes para instituição da Internação Domiciliar no âmbito do SUS. Brasília (DF): Ministério da Saúde; 2006.

17. Conselho Nacional de Saúde (Brasil). Resolução 196/ 96, de 10 de outubro de 1996. Dispõe sobre as diretrizes e normas regulamentadoras de pesquisa envolvendo seres humanos. Brasília (DF): Ministério da Saúde; 1996.

18. Almeida TL. Características dos cuidadores de idosos dependentes no contexto da Saúde da Família. [monografia on-line].. Ribeirão Preto: Universidade de São Paulo, Faculdade de Medicina de Ribeirão Preto; 2005.

19. Perlini NMOG, Faro ACM. Cuidar de pessoa incapacitada por acidente vascular cerebral no domicílio: o fazer do cuidador familiar. Rev Esc Enferm USP 2005; 39(2):154-63.

20. Bettega RTC, Bozza SM, Kranski ET. Perfil do atendimento domiciliar ao paciente oncológico fora de possibilidades terapêuticas no HEG. Prática Hospitalar 2005; 7(41).

21. Lacerda MR, Oliniski SR, Giacomozzi CM. Familiares cuidadores comparando a internação domiciliar e a hospitalar. Revista Família Saúde Desenvolvimento 2004;6(2):110-8. 


\section{Abstract}

This study aimed to identify the social profile of oncology patient caregivers assisted by the Palliative Care Team of the Home Care System of Londrina (PR) between January and July 2008, as well as review how healthcare is prepared and activities are developed by caregivers. The study sample consisted of 35 caregivers and the data were processed and analyzed with the Statistical Package for the Social Sciences (SPSS) software, version 11.5 for Windows. $85.7 \%$ of the caregivers were women whose average age was 53 . Most of the caregivers had children $(88.6 \%)$; were Evangelical $(48.6 \%)$ or Catholic $(45.7 \%)$; had a life partner $(77.1 \%)$, a job $(60.0 \%)$ and had between $1-4(37.1 \%)$ or $9-11(34.3 \%)$ years of schooling. They became caregivers due to lack of another option $(42.8 \%)$ or by their own choice $(40.0 \%)$. They had been working in the field for an average of 18 months. Their service included heavy lifting $(88.5 \%)$ specific care tasks $(80.0 \%)$ and related domestic activities for the patients (94.2\%). 85.7\% suffered physical pain and $45.7 \%$ had moments of recreation. $51.4 \%$ preferred patient death would occur at home. They felt prepared both to give care $(74.3 \%)$ and to deal with patient death at home $(57.2 \%)$. The in-home caregiver felt prepared for both domestic care and death, despite performing heavy-duty and complex services besides domestic services, feeling body pain and having few leisure activities. To understand the characteristics of these workers is fundamental to design and carry out qualified in-home palliative care.

Key words: Caregivers; Social Conditions; Hospice Care; Home Nursing; Medical Oncology; Londrina city

\section{Resumen}

Este estudio tuvo como objetivo identificar el perfil social de los cuidadores de pacientes oncológicos asistidos por el equipo de Cuidados Paliativos del Sistema de Internación Domiciliaria de Londrina (PR) de enero a julio de 2008, así como la revisión de la preparación para el cuidado y las actividades desarrolladas por los cuidadores. La muestra estuvo constituida de 35 cuidadores y los datos fueron procesados y analizados en el programa Statistical Package for the Social Science (SPSS) version 11.5 for Windows. Entre los cuidadores, el 85,7\% eran mujeres con una edad media de 53 años. La mayoría tenía hijos $(88,6 \%)$; era evangélica $(48,6 \%)$ o católica (45,7\%); tenía una pareja (77,1\%); poseía una ocupación (60\%) y tenía entre $1-4$ (37,1\%) o 9-11 (34,3\%) años de estudio. Se convirtieron cuidadores por falta de otra opción $(42,8 \%)$ o por elección propia $(40,0 \%)$. Se hicieron cargo de la función promedio durante 18 meses. Prestaban cuidados pesados 88,5\%, cuidados específicos $80 \%$ y las actividades domésticas vinculadas a los cuidados $94,2 \%$ de los cuidadores. Sentían dolor físico $85,7 \%$ y $45,7 \%$ tenían momentos de recreación. La mayoría de los cuidadores manifestó preferencia por la muerte del paciente en el hogar (51,4\%). Estaban preparados para cuidar, $74,3 \%$ de los cuidadores, y para la muerte en el hogar $57,2 \%$. El cuidador domiciliario se siente preparado para el cuidado y la muerte en el hogar, a pesar de realizar cuidados pesados y específicos, además de los domésticos; sentir dolor físico y realizar pocas actividades recreativas. Conocer a ese personaje es fundamental para la planificación de las acciones y ejecución de cuidados paliativos de calidad en el hogar.

Palabras clave: Cuidadores; Condiciones Sociales; Cuidados Paliativos; Atención Domiciliaria de Salud; Oncología Médica; Londrina (PR) 